\title{
Coagulation and fibrinolytic markers in a two-month follow-up of coronary bypass surgery
}

\author{
Alessandro Parolari, MD, $\mathrm{PhD}^{\mathrm{a}}$ \\ Susanna Colli, $\mathrm{PhD}^{\mathrm{b}}$ \\ Luciana Mussoni, $\mathrm{PhD}^{\mathrm{b}}$ \\ Sonia Eligini, $\mathrm{PhD}^{\mathrm{b}}$ \\ Moreno Naliato, $\mathrm{MD}^{\mathrm{a}}$ \\ Xin Wang, $\mathrm{MD}^{\mathrm{a}}$ \\ Sara Gandini, $\mathrm{MSc}^{\mathrm{C}}$ \\ Elena Tremoli, PhD ${ }^{a, b}$ \\ Paolo Biglioli MD \\ Francesco Alamanni, MD
}

\footnotetext{
From the Department of Cardiac Surgery, Centro Cardiologico Monzino IRCCS, ${ }^{\mathrm{a}} \mathrm{De}-$ partment of Pharmacological Sciences, E. Grossi Paoletti Center, University of Milan, ${ }^{\text {b }}$ and Department of Biostatistics, European Institute of Oncology, ${ }^{\mathrm{c}}$ Milan, Italy.

This work was supported in part by a grant from the Italian Ministry of Health (ICS 030.6 RF99.14).

Received for publication Feb 14, 2002; revisions requested June 13, 2002; revisions received June 27, 2002; accepted for publication July 15, 2002.

Address for reprints: Alessandro Parolari, $\mathrm{MD}, \mathrm{PhD}$, Department of Cardiac Surgery, University of Milan, Centro Cardiologico, Fondazione Monzino IRCCS, Via Parea, 4 , 20138, Milan, Italy (E-mail: alessandro.parolari@cardiologicomonzino.it J Thorac Cardiovasc Surg 2003;125:336-43

Copyright $(\odot 2003$ by The American Association for Thoracic Surgery

$0022-5223 / 2003 \$ 30.00+0$

doi: $10.1067 / \mathrm{mtc} .2003 .2$
}

Objectives: The alterations of the coagulation-fibrinolytic profile immediately and up to few days after cardiac surgery have been widely documented. However, less information is available on whether these alterations persist for prolonged periods of time after the operation. In this study we have evaluated the coagulation-fibrinolytic profile of patients who underwent coronary artery surgery with cardiopulmonary bypass during a 2-month follow-up period.

Methods: Twenty-six patients (age range, 50-75 years) were studied. Blood samples were collected before the intervention and at different time points postoperatively up to 2 months after the operation. Measurement of selected coagulation-fibrinolytic variables was carried out in plasma from 16 patients. Evaluation of tissue factor activity determined as procoagulant activity was performed in peripheral blood mononuclear leukocytes obtained from 10 patients.

Results: Antigenic levels of clottable fibrinogen, prothrombin fragment F1.2, Ddimer, and thrombin-antithrombin complex were significantly increased during the first week after the intervention compared with preoperative values. Prothrombin fragment F1.2 levels returned to normal within 15 days, fibrinogen levels normalized within 30 days, and thrombin-antithrombin complex levels normalized at 45 days, whereas D-dimer values were still significantly higher 60 days postoperatively respective to baseline values. There was a trend toward an increased procoagulant activity from peripheral blood mononuclear leukocytes 4 days after the operation, whereas no changes of factor VII measured either as antigen or in its coagulant and activated forms were recorded throughout the study.

Conclusions: A marked activation of the coagulation-fibrinolytic system occurs after cardiopulmonary bypass and lasts for at least 2 months thereafter. This finding suggests that these alterations might account for the increased thrombotic risk of these patients during the postoperative period.

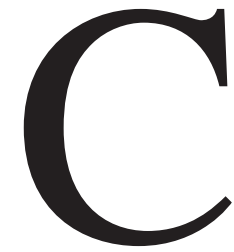
ardiac surgery with cardiopulmonary bypass $(\mathrm{CPB})$ can alter normal hemostatic balance and predispose patients either to immediate risk of excessive bleeding or to later thrombotic complications, such as ischemic events or early graft occlusion. ${ }^{1,2}$ Moreover, activation of both the fibrinolytic and inflammatory pathways can contribute to the so-called postpump syndrome, which is characterized by an impairment of myocardial, cerebral, pulmonary, and renal function. ${ }^{3}$ 
Early activation of coagulation, as shown by the increased levels of prothrombin fragment F1.2 and thrombinantithrombin complex (TAT), has been documented in patients who underwent CPB during cardiac surgery. ${ }^{4-6}$ Activation of the fibrinolytic system follows. Indeed, increased levels of D-dimer (XDP) ${ }^{7}$ and plasmin-antiplasmin complex ${ }^{4}$ have been reported as a consequence of the activation of the coagulation system.

Activation of coagulation recognizes multiple pathways that are related to various aspects of the intervention, such as surgical trauma, interaction of blood with extensive nonendothelial surfaces of the bypass circuit, and retransfusion of pericardial blood.6,8 More recently, tissue factor (TF) expressed by monocytes during and immediately after the procedure has been reported to play a pivotal role in CPBinduced coagulopathy. ${ }^{6,9}$ The end point of both intrinsic and extrinsic coagulation pathways is indeed the generation of thrombin, the prominent enzyme in the activation of hemostasis.

The behavior of hemostatic variables immediately after and in the time frame that spans from a few hours to 2 to 3 days after cardiac surgery with CPB is widely documented. ${ }^{8}$ On the contrary, less information is available about the behavior of these variables in a longer follow-up period. The lack of information is due to the fact that patients with uncomplicated procedures are usually discharged from the hospital 4 to 8 days after the operation, making it difficult to recall them over time.

Previous studies from our group and others ${ }^{10-12}$ have reported increased thrombin generation and alteration of fibrinolytic activity in patients undergoing CPB that lasted up to 8 to 30 days after the intervention and that were not prevented by the administration of aprotinin during the procedure. ${ }^{10}$

On this basis, it might be hypothesized that cardiac surgery with $\mathrm{CPB}$ activation of the coagulation-fibrinolytic pathways occurs not only during the operation but also later. The goal of the present study was therefore to assess whether alterations of the coagulation persist for longer follow-up periods. Moreover, the contribution of TF from mononuclear leukocytes in the occurrence of activation of coagulation within the first week after the intervention has been investigated.

\section{Patients and Methods}

\section{Patients}

Twenty-six patients were consecutively enrolled among those undergoing CPB for elective coronary artery bypass graft surgery, according to the American Heart Association/American College of Cardiology guidelines. ${ }^{13}$ Preoperative ejection fraction was greater than $30 \%$, and left ventricular end-diastolic pressure was less than $20 \mathrm{~mm} \mathrm{Hg}$. In order to avoid excessive blood sampling from the same subject, coagulation/fibrinolytic variables were measured in 16 patients whereas blood from 10 patients was processed for peripheral blood mononuclear leukocyte (PBML) isolation. All patients provided informed consent to participate in this study that was approved by the Ethical Committee of Centro Cardiologico Monzino IRCCS. None of the patients had renal or liver dysfunction or were taking drugs affecting coagulation-fibrinolytic variables.

\section{Surgical Procedure}

All patients underwent intervention by the same surgical team. On the morning of the operation, patients received their usual dose of antianginal drugs, with 2 to $5 \mathrm{mg}$ of morphine and $1 \mathrm{mg}$ of atropine as premedication. Anesthesia was induced by the administration of sodium thiopental (3 mg/kg), fentanyl $(0.75 \mu \mathrm{g} / \mathrm{kg})$, succinylcholine $(1 \mathrm{mg} / \mathrm{kg})$, diazepam $(10 \mathrm{mg})$, and pancuronium bromide $(0.1$ $\mathrm{mg} / \mathrm{kg}$ ). After endotracheal intubation, patients were ventilated to normocapnia with an oxygen and air mixture. Boluses of fentanyl (with or without droperidol), diazepam, and pancuronium bromide were given when necessary. Cefuroxime ( $2 \mathrm{~g}$ ) was given intravenously for infection prophylaxis. A radial artery catheter and a flow-directed pulmonary artery catheter (Swan-Ganz, Baxter/ American Edwards Laboratories) were inserted for hemodynamic measurements. The extracorporeal circuit consisted of a roller pump, hollow-fiber oxygenator with integrated heat exchanger, arterial filter, open cardiotomy reservoir, and polyvinyl tubing system. The extracorporeal circuit was primed with $1500 \mathrm{~mL}$ of crystalloid solution and $5000 \mathrm{IU}$ of bovine lung heparin. After systemic heparinization (300 IU/kg bovine lung heparin), CPB was initiated by the placement of cannulas in the ascending aorta and right atrium (2-stage venous cannula). Activated clotting time was kept at 440 seconds or greater with additional heparin. The nonpulsatile flow rate was maintained at $2.4 \mathrm{~L} \times \min ^{-1} \times \mathrm{m}^{-2}$ during the cooling and rewarming phases and at $2.0 \mathrm{~L} \times \min ^{-1} \times \mathrm{m}^{-2}$ during stable hypothermia. Before and after CPB, blood from mediastinum was collected with a cell-saver device, washed, and returned to the patient, whereas during $\mathrm{CPB}$, mediastinal blood was returned directly to the extracorporeal circuit. Mean arterial pressure was kept at 50 to $90 \mathrm{~mm} \mathrm{Hg}$. Patients were cooled to $29^{\circ} \mathrm{C}$ to $31^{\circ} \mathrm{C}$ and received a first dose $(1000 \mathrm{~mL})$ of cool $\left(4^{\circ} \mathrm{C}\right.$ antegrade and retrograde high-potassium crystalloid cardioplegia (St Thomas Hospital II Cardioplegic Solution, SALF) just after aortic crossclamping. This maneuver was repeated (250 $\mathrm{mL}$ retrogradely) every 20 minutes of aortic crossclamp time. Disturbances in the acid-base balance were appropriately treated, and acid-base equilibrium was maintained by using the alpha-stat method. The hematocrit level was maintained within $18 \%$ to $25 \%$ throughout the procedure.

After termination of the intervention, heparin was antagonized with protamine sulfate at a $1: 1$ ratio $(3 \mathrm{mg} / \mathrm{kg})$. None of the patients required inotropic support for weaning from CPB. After the operation, patients were admitted to the intensive care unit (ICU) and treated according to a standardized protocol. Mean arterial blood pressure was kept at 70 to $90 \mathrm{~mm} \mathrm{Hg}$, heart rate at 70 to 90 beats/min, and cardiac index at greater than 2.0 $\mathrm{L} \times \min ^{-1} \times \mathrm{m}^{-2}$. Patients were ventilated to normocapnia, and an arterial oxygen tension of $80 \mathrm{~mm} \mathrm{Hg}$ with continuous positivepressure ventilation and positive end-expiratory pressure of $5 \mathrm{~cm}$ $\mathrm{H}_{2} \mathrm{O}$ was maintained until extubation, according to the ICU regimen. Basic fluid administration consisted of $0.9 \% \mathrm{NaCl}$ and poly- 
gelatine. Packed erythrocytes were infused when the hematocrit level was less than $18 \%$ during CPB and less than $24 \%$ in the ICU. When the cardiorespiratory condition had stabilized, patients were transported to the ward for further recovery. In the postoperative period, patients received subcutaneous heparin (5000 IU twice daily), starting from 24 hours to 72 hours after CPB, followed by aspirin (325 mg/day), which was usually given within the first and second days after the intervention.

\section{Follow-up}

All the patients were hospitalized until the eighth postoperative day. Sixteen of them had periodic follow-up visits (physical examination, electrocardiography, and blood collection for coagulation-fibrinolytic variables) at the 15th postoperative day and every 15 days on completion of the study protocol at the 60th postoperative day.

\section{Blood Sampling}

Blood collection was performed from a peripheral vein through a 19 -gauge needle in plastic tubes $(0.13 \mathrm{~mol} / \mathrm{L}$ sodium citrate or 15 $\mathrm{U} / \mathrm{mL}$ heparin as an anticoagulant, see below) during the morning. Blood for measurement of coagulation-fibrinolytic variables was collected at the following time points: the day before the operation $(-1)$ and the 4th $(+4), 8$ th $(+8), 15$ th $(+15), 30$ th $(+30), 45$ th $(+45)$, and 60th $(+60)$ postoperative day. Blood for mononuclear leukocyte isolation was collected the day before the operation $(-1)$ and at the fourth $(+4)$ and eighth $(+8)$ postoperative day.

\section{Laboratory Methods}

For selected coagulation-fibrinolytic variable measurements, plasma was prepared by means of centrifugation at $1500 \mathrm{~g}$ for 20 minutes at $4^{\circ} \mathrm{C}$ from blood collected in $0.13 \mathrm{~mol} / \mathrm{L}$ sodium citrate ( $1: 10 \mathrm{vol} / \mathrm{vol})$ within 1 hour after venipuncture. Centrifugation was carried out at room temperature for factor VII determination. Plasma was divided into aliquots and frozen at $-80^{\circ} \mathrm{C}$ until assay.

Fibrinogen levels were measured according to the method of Clauss ${ }^{14}$ (Dade-Thrombin, Dade Diagnostics) with a coagulometer (Schnitger Gross).

TAT, XDP, and factor VII antigen levels were determined by using ELISA with commercially available kits, according to the manufacturers' recommendations (Enzygnost TAT, Dade Behring; Dimer-test Gold Stripwell EIA kit, Instrumentation Laboratory; and Asserachrom VII:ag, Stago Boehringer). Prothrombin fragment F1.2 was assayed in heparinized plasma by using a commercially available ELISA kit (Enzygnost F1.2, Dade Behring). Plasma factor VII coagulant was assessed by using a 1-stage clotting time assay with a coagulometer, with factor VII-deficient plasma and human tissue thromboplastin as reagents (Instrumentation Laboratory). Plasma factor VII activity was measured by using a 1-stage clotting assay with a soluble mutant recombinant TF (kind gift from J. H. Morrissey, University of Illinois) that possesses cofactor activity for factor VII activity but fails to support activation of factor VII. ${ }^{15}$ Clotting times were converted to factor VII activity concentration (in nanograms per milliliter) by means of comparison with a standard curve of purified recombinant factor VII activity (National Institute of Biological Standards and Control, United Kingdom, code 89/688). Coagulation-fibrinolytic data were normalized for hematocrit values.

\section{Blood Sampling for PBML Isolation}

Blood was drawn in sterile tubes containing heparin $(10 \mathrm{U} / \mathrm{mL})$ and divided into 2 aliquots under sterile conditions, according to the method of Osterud and Bjorklid. ${ }^{16}$ Saline solution or $2 \mathrm{ng} / \mathrm{mL}$ bacterial lipopolysaccharide (LPS; Sigma) was added, and samples were incubated at $37^{\circ} \mathrm{C}$. Reaction was stopped after 2 hours by adding ethylenediamine tetra-acetic acid (2\%, pH 7.4). Plateletrich plasma was separated (2300 rpm for 4 minutes) and replaced with culture medium RPMI (BioWhittaker Italia) containing sodium citrate. PBMLs were obtained by means of sedimentation on Ficoll-Paque (Pharmacia) density gradient. Cells were washed, resuspended in PBS, and diluted to $2 \times 10^{6} / \mathrm{mL}$. Aliquots $(100$ $\mu \mathrm{L})$ of cell suspensions were subjected to repeated cycles of freezing and thawing and used to assess procoagulant activity.

\section{Assay of Procoagulant Activity}

Procoagulant activity was measured on cell lysates by using the plasma recalcification time assay. In brief, citrated plasma (100 $\mu \mathrm{L})$ was incubated with cell lysate $(100 \mu \mathrm{L})$, and $25 \mathrm{~mol} / \mathrm{L} \mathrm{CaCl}_{2}$ was added to initiate the reaction. Clotting times were converted into arbitrary units by using reference curves determined with a standard human placental thromboplastin preparation (Thromborel Behring). The logarithm of the clotting times was related to the logarithm of the procoagulant activity. Experiments performed with factor VII-deficient plasma verified that the procoagulant activity measured was TF activity. Data are therefore expressed as units of TF activity per milliliter, and the TF activity ratio of LPS-stimulated/LPS-unstimulated cells is reported.

\section{Statistical Analysis}

Clinical data of patients are reported as means $\pm 1 \mathrm{SD}$ or as percentages. Trends by time in prothrombin fragment F1.2, TAT, fibrinogen, XDP, factor VII (as antigen or in its coagulant and activated forms), and TF activity were studied by using a repeated measurements analysis, taking into account the correlation among the measurements at the different time periods. A variance component correlation structure was used, and there was no evidence that a more complex structure was needed. Contrasts were calculated to estimate the mean change of the different variables from baseline. The normality assumptions were checked by using normal plots, and logarithm transformation was used to achieve normality for prothrombin fragment F1.2, TAT, and XDP. One subject was excluded from the analysis because of TAT and prothrombin fragment F1.2 preoperative values exceeding 2 SDs.

Fractional polynomials were fitted to find suitable models for the curved relationship between the different variables and time. ${ }^{17}$ In the graphs the predicted values from these models, as well as the observed values for each subject by time, are plotted. All calculations were performed by using PROC MIXED and PROC GLM in SAS software (SAS for Windows version 6.11, SAS Institute Inc).

\section{Results}

\section{Clinical Results}

Clinical variables of the patients are reported in Table 1. None of the patients underwent re-exploration for bleeding, 
and the postoperative course was uneventful and uncomplicated for all patients.

\section{Coagulation-fibrinolytic Variables}

Analysis of variance for repeated measures showed a significant time effect for the variables prothrombin fragment F1.2 $(P=.0004)$, TAT $(P<.0001)$, fibrinogen $(P<$ $.0001)$, and XDP $(P<.0001)$ and a trend toward a significant effect of time for TF activity $(P=.06)$ expressed as the ratio of LPS-stimulated/LPS-unstimulated activity. Factor VII as antigen and in its coagulant and activated forms did not show significant variations over time $(P>.1)$.

\section{Prothrombin Fragment F1.2}

Prothrombin fragment F1.2 levels increased significantly from baseline $(0.35 \pm 0.216 \mathrm{nmol} / \mathrm{L})$ at the fourth and eighth day postoperatively. The increase from baseline values, as estimated from percentage changes on the leastsquared means obtained from repeated measurements analysis, was $95.3 \%$ (95\% confidence interval [CI], 38.5-175.5) and $57.4 \%$ (95\% CI, 12.2-120.7) at the fourth and eighth day, respectively. Thereafter, these levels decreased, with the estimated percentage change being $27.8 \%$ greater than the baseline value 15 days after the intervention, with a complete return to baseline values occurring at the 45th postoperative day (Figure 1).

\section{Thrombin-antithrombin complex}

TAT levels increased from the baseline point $(3.8 \pm 2.19$ $\mathrm{ng} / \mathrm{mL}$ ) starting the fourth day after the operation and reached its peak 15 days after the intervention, with a percentage change estimated on the least-squared means equal to $204.1 \%$ (95\% CI, 95.1-374.1; Figure 2); TAT levels remained significantly higher than baseline values up to 30 days after the intervention ( $+96.7 \%$; 95\% CI, 27.1204.4). A return to preoperative levels was detected at the 45th postoperative day.

\section{Fibrinogen}

Fibrinogen levels markedly increased from baseline (359 \pm $88 \mathrm{mg} / \mathrm{dL})$ at the fourth postoperative day $(+103.0 \%$; $95 \%$ CI, 73.8-137.1), remained significantly increased up to the 15 th day $(+52.7 \%$; $95 \%$ CI, 31.1-77.9), and returned to preoperative levels within the 45th postoperative day (Figure 3).

\section{XDP}

XDP levels markedly and progressively increased from baseline $(0.044 \pm 0.083 \mu \mathrm{g} / \mathrm{mL})$ up to the 15 th day after CPB (+9979.3\%; 95\% CI, 3291.6-29853.9). Then the levels decreased, although remaining significantly higher respective to baseline values even at the 60th day $(+418.8 \%$; 95\% CI, 74.6-1441.9; Figure 4).
TABLE 1. Clinical variables of the patient population $(n=$ 26)

\begin{tabular}{lc}
\hline Age (y) & $59 \pm 5$ \\
BSA (m²) & $1.88 \pm 0.19$ \\
Male sex & $24(92 \%)$ \\
Previous MI & $15(60 \%)$ \\
Stable angina & $26(100 \%)$ \\
Angiographic EF & $56.9 \pm 7.9$ \\
Distal anastomoses & $3.4 \pm 0.6$ \\
LITA use & $26(100 \%)$ \\
CPB time (min) & $84 \pm 17$ \\
Xclamp time (min) & $64 \pm 10$ \\
24-h bleeding (mL) & $662 \pm 352$ \\
Re-exploration for bleeding & 0
\end{tabular}

$\overline{B S A}$, Body surface area; $E F$, ejection fraction; LITA, left internal thoracic artery; MI, myocardial infarction; Xclamp, aortic crossclamp.

\section{Factor VII}

No changes of factor VII activity and antigen and the activated factor VII were detected throughout the postoperative period (not shown).

\section{TF Activity}

Before the operation, freshly isolated PBMLs expressed $1.44 \pm 0.84 \mathrm{U} / \mathrm{mL}$ TF activity, and this increased to $5.01 \pm$ $5.8 \mathrm{U} / \mathrm{mL}$ on exposure to $2 \mathrm{ng} / \mathrm{mL}$ LPS. TF activity in unstimulated cells did not change postoperatively, whereas it increased up to $8.05 \pm 6.9 \mathrm{U} / \mathrm{mL}$ at the fourth postoperative day. TF activity expressed as the ratio of LPS-stimulated/LPS-unstimulated PBMLs showed a trend $(P=.06)$ toward a significant increase at the fourth postoperative day respective to preoperative values (Figure 5).

\section{Discussion}

Previous studies have shown that the early period after CPB procedures is the period of highest risk for thrombotic complications. The highest rate of bypass occlusions occurs within the first months after CPB. During this period of time, the percentage of patients who experience occlusion of at least one graft is between $3 \%$ and $12 \%$ after 1 month, ${ }^{18}$ and this value can increase further 3 months after the operation. ${ }^{19}$ In addition to the problem of graft occlusions, other complications on a thrombotic basis can frequently occur. The incidence rates reported for perioperative stroke are usually from $2 \%$ to $3 \%,{ }^{20}$ reaching $5 \%$ in some reports. ${ }^{21}$ The majority of strokes occur, however, in a time frame up to 10 days after surgical intervention, after awakening from surgical intervention, or after an uneventful neurologic recovery. ${ }^{21}$ This observation suggests that factors other than those related to the complexity of the intervention might contribute to the occurrence of thrombotic events.

Our results indicate that alterations of the coagulationfibrinolytic profile, which are markedly evident within 4 to 


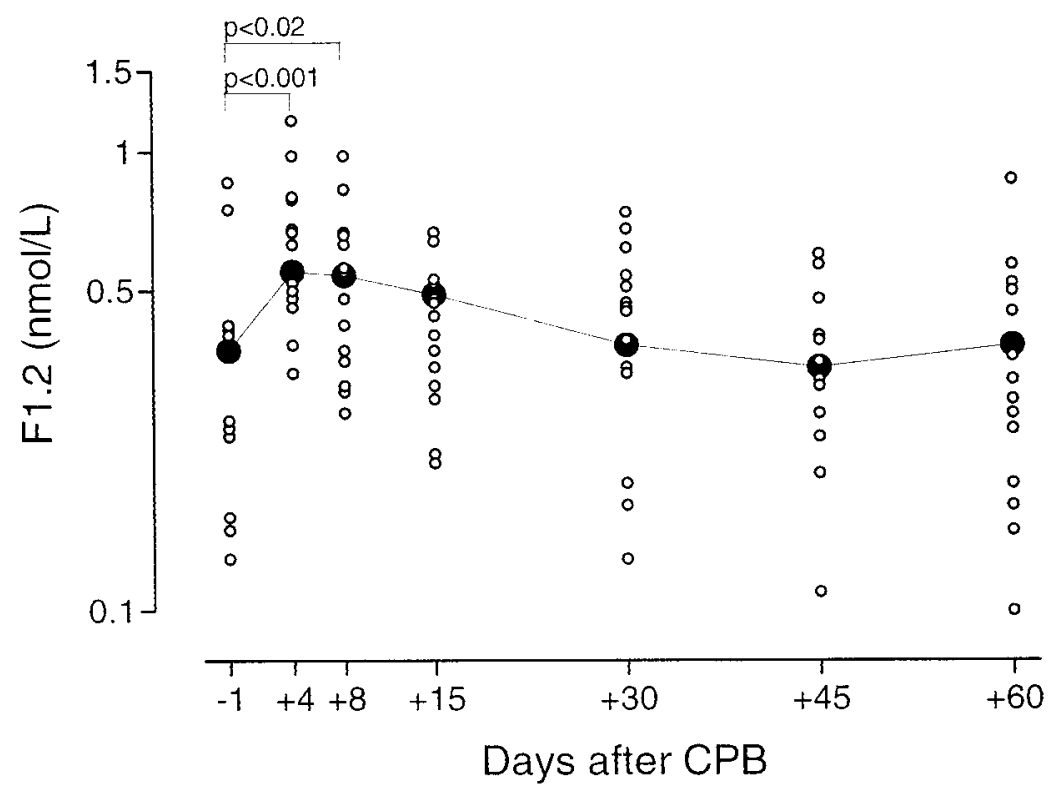

Figure 1. Plasma levels of prothrombin fragment F1.2 preoperatively and up to the 60th postoperative day. Small open circles represent observed values, and large filled circles represent fitted values from the statistical model.

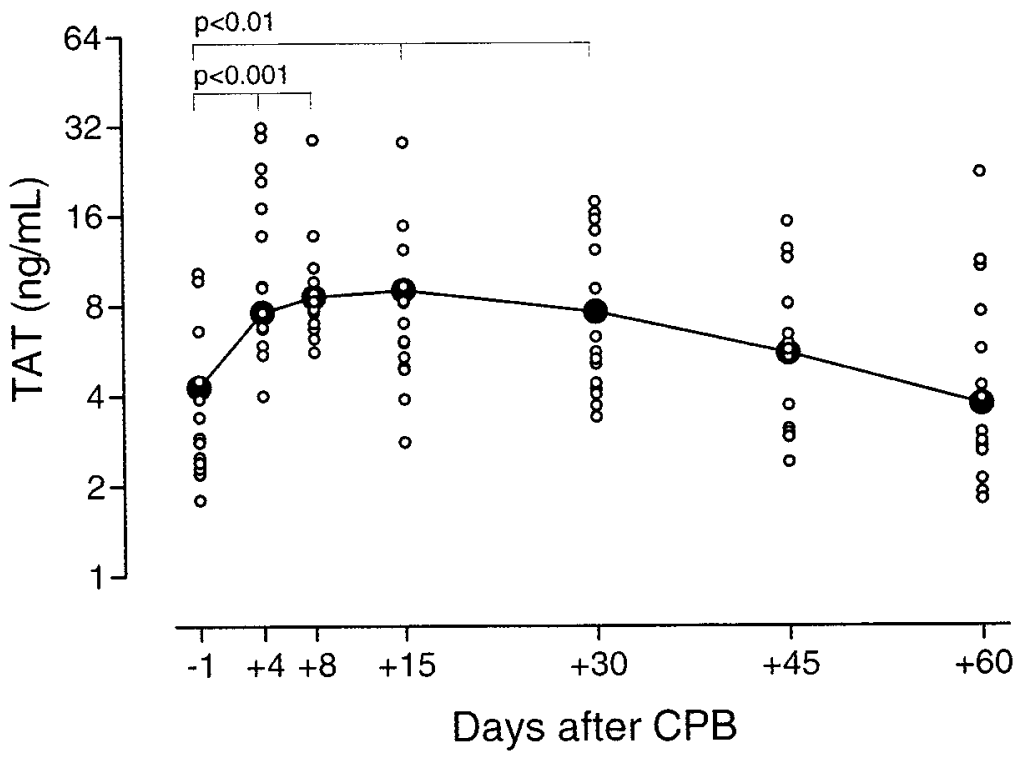

Figure 2. Plasma levels of TAT preoperatively and up to the 60th postoperative day. Small open circles represent observed values, and large filled circles represent fitted values from the statistical model.

8 days from the intervention, persist for at least 2 months of the follow-up period. In more detail, fibrinogen levels were significantly higher at the 15th postoperative day, and TAT and XDP levels remained higher at the 30th and 60th postoperative days, respectively. This latter finding suggests that 2 months after the operation, the generation of plasmin still persists, both as a consequence of ongoing removal of hemostatic plugs and formation of uncovered intravascular fibrin deposition. D-dimer is in fact recognized as an indicator of fibrin formation in vivo. ${ }^{22}$ Moreover, as suggested by others, ${ }^{23}$ increased levels of XDP might result from a remodeling of clot in the operative site.

Up to now, only one study has evaluated the hemostaticprothrombotic profile after CPB during a relative long-term follow-up (3 months). ${ }^{12}$ In this study TAT and fibrinogen levels that were found to be significantly increased at the 


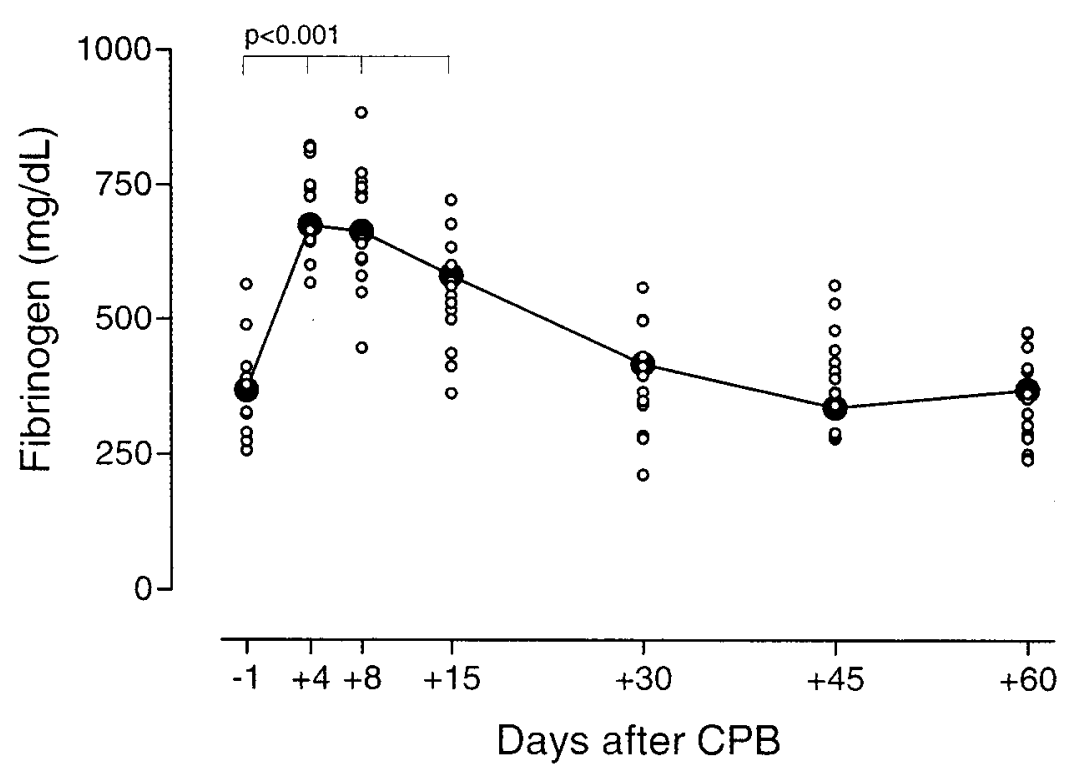

Figure 3. Plasma levels of fibrinogen preoperatively and up to the 60th postoperative day. Small open circles represent observed values, and large filled circles represent fitted values from the statistical model.

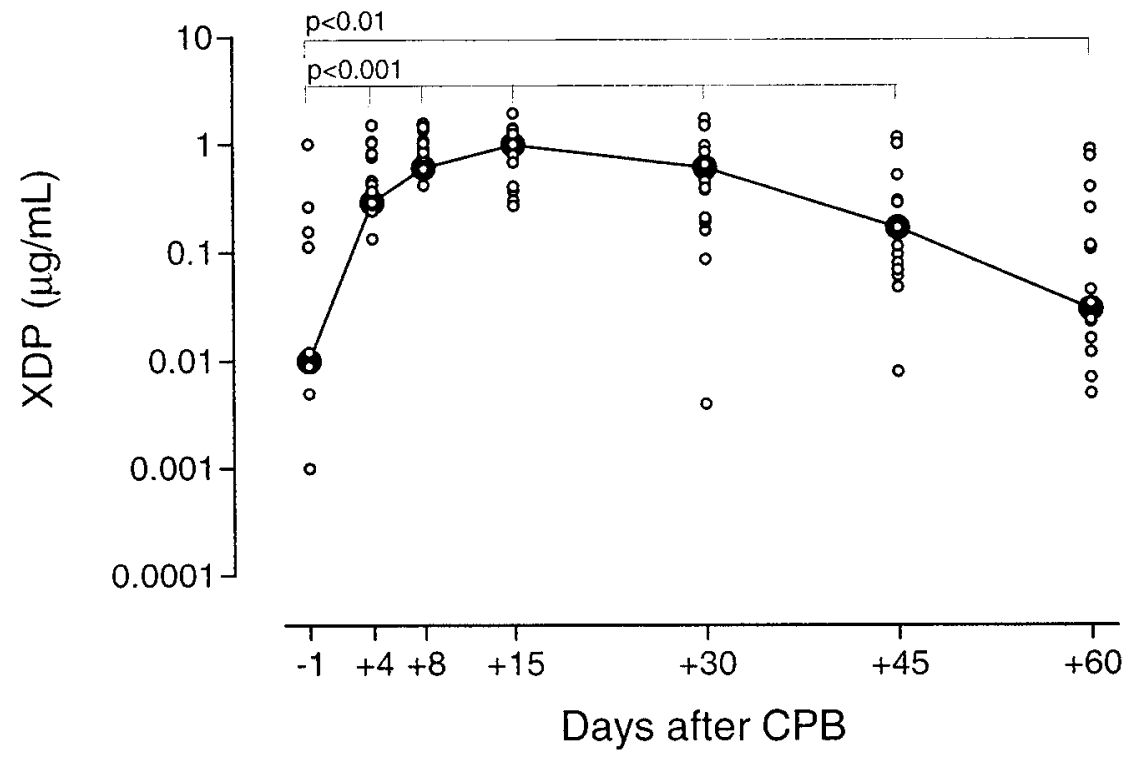

Figure 4. Plasma levels of XDP preoperatively and up to the 60th postoperative day. Small open circles represent observed values, and large filled circles represent fitted values from the statistical model.

eighth day normalized after 3 months. Results from our study document that thrombin generation is still increased 2 months after the operation.

A trend toward increase of TF activity by PBMLs was detected 4 days postoperatively. This increase, regardless of a large interindividual variability, indicates that monocyte activation in terms of TF activity likely persists for at least some days after the operation.

The extracorporeal circuit has been previously shown to be critical in terms of monocyte activation. Indeed, Barstad and colleagues ${ }^{9}$ showed an increase of TF in adherent monocytes retrieved from the oxygenators immediately after bypass arrest that was reduced when heparin-coated circuits and systemic heparinization were adopted. Moreover, as far as PBMLs are considered, alterations of the extrinsic pathway of coagulation have been documented during and soon after cardiac surgery performed with the use of CPB. ${ }^{5,24}$ Ernofsson and associates ${ }^{24}$ reported that TF 


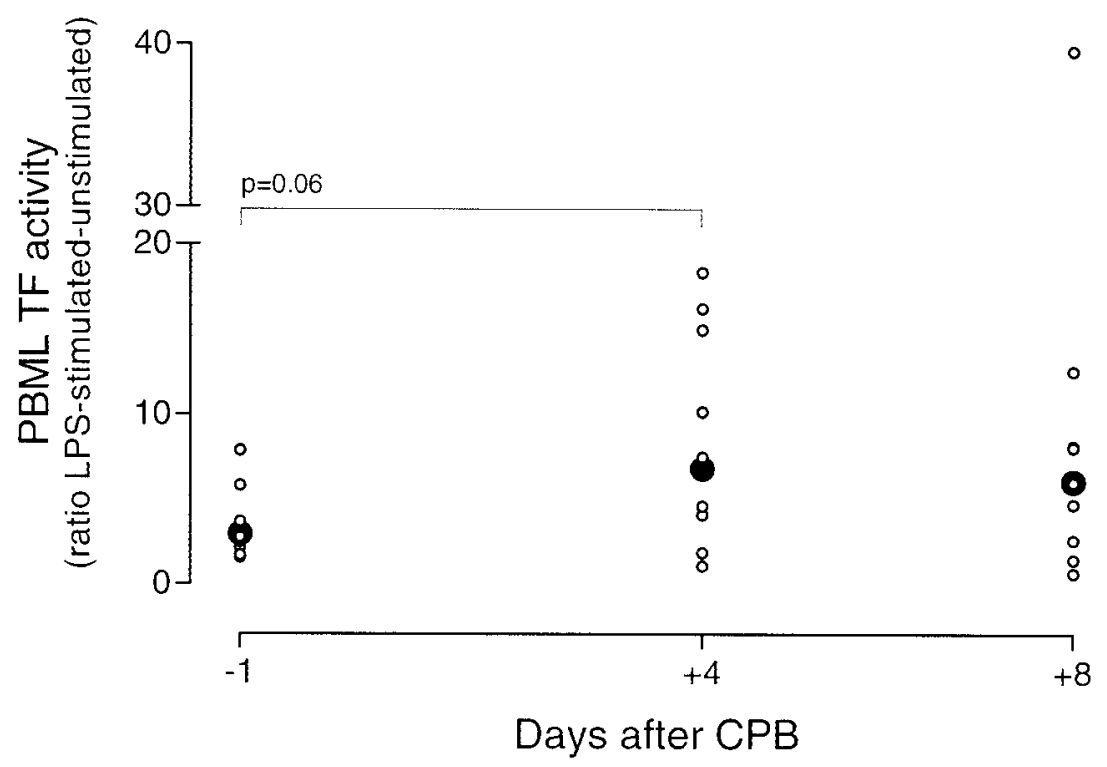

Figure 5. TF activity by PBMLs (expressed as ratio of LPS-stimulated/LPS-unstimulated cells) preoperatively and up to the eighth postoperative day. Small open circles represent observed values, and large filled circles represent fitted values from the statistical model.

expression was not changed at the end of $\mathrm{CPB}$, whereas a significant increase of TF was recorded 20 hours after the operation. Interestingly, the same authors documented such an increase both in unstimulated monocytes and in monocytes exposed to LPS. These data allowed the conclusion that TF expression in circulating monocytes is upregulated the day after heart operation. Our results extend this observation and suggest that relatively increased levels of TF activity in the systemic circulation might persist longer after the operation, thus addressing a role of TF as a generator of thrombin not only during and immediately after the intervention but also in a more prolonged follow-up period.

Previous data by Nieuwland and coworkers ${ }^{25}$ showed the presence of higher levels of procoagulant cell-derived microparticles in pericardial blood from patients undergoing $\mathrm{CPB}$ with respect to systemic circulation. The finding about an increased procoagulant profile of the blood collected from the pericardial cavity respective to systemic circulation was also confirmed by a more recent study. ${ }^{26}$ These results, however, have been obtained either after the release of the aortic clamp (from 5-10 minutes) or in a time frame from 15 to 60 minutes on bypass, respectively. In the latter condition, plasma TF from the systemic circulation was not increased, thus reflecting a different behavior between cells involved in the surgical damage and those from peripheral blood $^{26}$ under the profile of time requested for TF to be exposed.

The observed upregulation of TF activity is strictly paralleled by activation of coagulation detected as an increase of prothrombin fragment F1.2 levels used as a marker for thrombin generation. The coagulopathy profile persists for longer time periods from the intervention, as documented by fibrinogen and TAT levels that are still increased 15 and 30 days postoperatively, respectively.

One limitation of this study is that no control group of patients undergoing another type of operation was studied; for this reason, this study cannot clearly address whether changes in coagulation-fibrinolytic variables are specific for cardiac surgery with CPB or common to all surgical procedures and wound healing. This issue is rather controversial. Indeed, it has been previously shown that coagulation and fibrinolytic variables are altered after some types of skeletal surgery ${ }^{27}$ and, in contrast, that a major activation of coagulation and fibrinolysis occurs after cardiac surgery performed with CPB but not after general thoracic surgery. ${ }^{7}$ Regardless of the different conclusions, it should be pointed out that the observation period considered in the above studies is limited to the first 24 to 48 hours after the operation. No information, however, is available about the behavior of the considered variables for longer periods of time.

At present, current indication for the prevention of cardiovascular events in patients undergoing $\mathrm{CPB}$ is to prescribe aspirin. ${ }^{18}$ Our observation of a persistent activation of coagulation 2 months after $\mathrm{CPB}$ raises the question of whether antithrombotic therapy should be reconsidered. However, this question needs to be answered on the basis of the results emerging from appropriately designed clinical trials. 


\section{References}

1. Spiess BD. Ischemia: a coagulation problem? J Cardiovasc Pharmacol. 1996;2(suppl 1):S38-41.

2. Smith RC, Leung JM, Mangano DT. Postoperative myocardial ischemia in patients undergoing coronary artery bypass graft. S.P.I. Research Group. Anesthesiology. 1991;74:464-73.

3. Edmunds LH. Inflammatory response to cardiopulmonary bypass. Ann Thorac Surg. 1998;66:S12-6.

4. Ovrum E, Brosstad F, Holen EA, Tangen G, Abdelnoor M. Effects on coagulation and fibrinolysis with reduced versus full systemic hepariniztion and heparin-coated cardiopulmonary bypass. Circulation. 1995;92:2579-84.

5. Boisclair MD, Lane DA, Philippou H, Esnouf MP, Sheikh S, Hunt B, et al. Mechanisms of thrombin generation during surgery and cardiopulmonary bypass. Blood. 1993;82:3350-7.

6. Chung JH, Gikakis N, Rao AK, Drake TA, Colman RW, Edmunds LH. Pericardial blood activates the extrinsic coagulation pathway during clinical cardiopulmonary bypass. Circulation. 1996;93:2014-8.

7. Hunt BJ, Parratt RN, Segal HC, Sheikh S, Kallis P, Yacoub M. Activation of coagulation and fibrinolysis during cardiothoracic operations. Ann Thorac Surg. 1998;65:712-8

8. Boyle EM, Verrier ED, Spiess BD. The procoagulant response to injury. Ann Thorac Surg. 1997;64:S16-23.

9. Barstad RM, Ovrum E, Ringdal MA, Oysters R, Hamers MJ, Veiby $\mathrm{OP}$, et al. Induction of monocyte tissue factor procoagulant activity during coronary artery bypass surgery is reduced with heparin-coated extracorporeal circuit. Br J Haematol. 1996;94:517-25

10. Mannucci L, Gerometta P, Mussoni L, Antona C, Parolari A, Salvi L, et al. One month follow-up of haemostatic variables in patients undergoing aortocoronary bypass surgery. Thromb Haemost. 1995;73: 356-61.

11. Davey FR, Parker FB. Delayed hemostatic changes following cardiopulmonary bypass. Am J Med Sci. 1976;271:171-8.

12. Moor E, Hamsten A, Blomback M, Herzfeld I, Wiman B, Ryden L. Hemostatic factors and inhibitors and coronary artery bypass grafting: preoperative alterations and relation to graft occlusion. Thromb Haemost. 1994;72:335-42.

13. Eagle KA, Guyton RA, Davidoff R, Ewy GA, Fonger J, Gardner TJ, et al. ACC/AHA guidelines and indications for coronary artery bypass graft surgery. A report of the American College of Cardiology/American Heart Association Task Force on Practice Guidelines. J Am Coll Cardiol. 1999;34:1262-346.
14. Clauss A. Gerinnungsphysiologische Schnell-Methode zur Bestimmung des fibrinogens. Acta Haematol 1957;17:237-46.

15. Morrissey JH, Fair DS, Edgington TS. Monoclonal antibody analysis of purified and cell-associated tissue factor. Thromb Res 1988;52:24761.

16. Osterud B, Bjorklid E. The production and availability of tissue thromboplastin in cellular populations of whole blood exposed to various concentrations of endotoxin: an assay for detection of endotoxin. Scand J Haematol 1982;29:175-84.

17. Royston P, Altman D. Regression using fractional polynomials of continuous covariates: parsimonious parametric modeling. Appl Stat. 1994;43:429-67.

18. Motwani JG, Topol EJ. Aortocoronary saphenous vein graft disease: pathogenesis, predisposition, and prevention. Circulation. 1998;97: 916-31.

19. Moor E, Blomback M, Silveira A, Wiman B, Cederlund K, Bergstrand $\mathrm{L}$, et al. Haemostatic function in patients undergoing coronary artery bypass grafting: preoperative perturbations and relations to saphenous vein graft closure. Thromb Res. 2000;98:39-49.

20. Taylor KM. Brain damage during cardiopulmonary bypass. Ann Thorac Surg. 1998;65(suppl 4):S20-6.

21. Hogue CW Jr, Murphy SF, Schechtman KB, Davila-Roman VG. Risk factors for early or delayed stroke after cardiac surgery. Circulation. 1999;100:642-7.

22. Dempfle CA. Use of D-dimer assays in the diagnosis of venous thrombosis. Semin Thromb Haemost. 2000;26:631-41.

23. Comunale ME, Carr JM, Moorman RM, Robertson LK. Significance of D-dimer concentrations during and after cardiopulmonary bypass. J Cardiothorac Vasc Anesth. 1996;10:477-81.

24. Ernoffson M, Thelin, Siegbahn A. Monocyte tissue factor expression, cell activation, and thrombin formation during cardiopulmonary bypass: a clinical study. J Thorac Cardiovasc Surg. 1997;113:576-84.

25. Nieuwland R, Berckmans RJ, Rotteveel-Eijkman RC, Maquelin KN, Roozendaal KJ, Jansen PGM, et al. Cell-derived microparticles generated in patients during cardiopulmonary bypass are highly procoagulant. Circulation. 1997;96:3534-41.

26. Philippou H, Adami A, Davidson SJ, Pepper JR, Burman JF, Lane DA. Tissue factor is rapidly elevated in plasma collected from the pericardial cavity during cardiopulmonary bypass. Thromb Haemost. 2000;84:124-8.

27. Pape HC, Schmidt RE, Rice J, van Griensven M, das Gupta R, Krettek $\mathrm{C}$, et al. Biochemical changes after trauma and skeletal surgery of the lower extremity: quantification of the operative burden. Crit Care Med 2000;28:3441-8.

Availability of Journal back issues

As a service to our subscribers, copies of back issues of The Journal of Thoracic and Cardiovascular Surgery for the preceding 5 years are maintained and are available for purchase from Mosby until inventory is depleted. Please write to Mosby, Subscription Customer Service, 6277 Sea Harbor Dr, Orlando, FL 32877, or call $800-654-2452$ or $407-345-4000$ for information on availability of particular issues and prices. 